\title{
Mandatory influenza vaccination? First we need a better vaccine
}

\author{
Michael Gardam MSc MDCM, Camille Lemieux BScPhm MD
}

I n light of the particularly severe 2012-2013 influenza season, great attention has been paid to influenza vaccination programs, including campaigns that target health care workers. In Canada, the vaccination of health care workers against influenza is almost exclusively voluntary; however, campaigns rarely achieve vaccination rates above $60 \% .^{1}$ This low uptake has led to increasing support in Canada for the mandatory annual vaccination of health care workers against influenza. ${ }^{2}$ An editorial supporting this approach was recently published in $C M A J$. $^{3}$

Some American organizations that require annual influenza vaccination as a condition of employment have reported vaccination rates of 95\% and higher. ${ }^{4}$ The message seems to be clear: the status quo of voluntary vaccination does not work, and mandatory vaccination does.

Are we comfortable that we have all of the evidence needed to move forward? We have certainly heard that influenza is responsible for up to 8000 deaths each year in Canada, ${ }^{5}$ and that the vaccination of health care workers decreases morbidity and all-cause mortality among residents of long-term care facilities and, by extrapolation, among all patients. ${ }^{2,6}$ These messages have spread from the pages of medical journals into the media and have become accepted as compelling truths. Until recently, however, few have gone back to the original studies on which these statements are based. We believe that it is of paramount importance to critically analyze the benefits of influenza vaccination before making a decision that justifies the suspension of a health care worker's right to refuse to be vaccinated.

Two research groups have recently reanalyzed the literature supporting influenza vaccination, including the vaccination of health care workers as a patient-safety measure. ${ }^{7,8}$ Both concluded that influenza vaccination is considerably less effective than is commonly accepted. These conclusions are not new: in 2007, Simonsen and colleagues ${ }^{9}$ similarly challenged the results of published influenza vaccine efficacy studies involving elderly patients. ${ }^{9}$

In their 2010 Cochrane review, Thomas and colleagues $^{7}$ evaluated 4 cluster randomized trials and 1 large cohort study, involving roughly
20000 patients over the age of 60 who lived in long-term care facilities, examining the effect of the vaccination of health care workers on specific influenza-related patient outcomes (laboratoryconfirmed influenza, pneumonia and pneumoniarelated deaths). They concluded that the vaccination of health care workers was not associated with a significant reduction in influenza-specific outcomes. However, pooled analysis of the 3 cluster randomized trials showed that vaccination of health care workers was associated with a significant reduction in nonspecific patient outcomes (influenza-like illness, all-cause mortality among patients $>60 \mathrm{yr}$ ). On the basis of a lack of an effect of vaccination on specific influenza-related outcomes, the authors concluded that there was insufficient evidence to support the vaccination of health care workers as a method of protecting patients in long-term care facilities.

Thomas and colleagues ${ }^{7}$ argue, and we agree, that influenza-specific mortality is a more valid measure of vaccine effectiveness. It can be challenging to determine whether influenza actually played a direct or indirect role in mortality, and, as per surveillance data from the Public Health Agency of Canada, most influenza-like illnesses in any given year are caused by other pathogens. ${ }^{10}$ Thomas and colleagues ${ }^{7}$ further argue that the discrepancy between influenza-specific and nonspecific outcomes likely stems from various confounders (i.e., differential uptake of the vaccine, varying attitudes of health care workers toward vaccination).

The recent systematic review by Osterholm and colleagues ${ }^{8}$ of the efficacy of the influenza

\section{KEY POINTS}

- The effectiveness of the current influenza vaccine has been exaggerated in the medical literature and media.

- A recent systematic review indicated that the efficacy and effectiveness of the influenza vaccine is about $60 \%$ in healthy adults.

- Despite mediocre effectiveness, it still makes sense for health care workers and others to receive the vaccine because there is very little downside to vaccination.

- Given the vaccine's considerable limitations, making vaccination mandatory is likely premature. 
vaccine found that, at best, the current influenza vaccine provides moderate (about $60 \%$ ) protection from laboratory-confirmed disease in healthy adults but that "such protection is greatly reduced or absent in some seasons." In a subsequent policy paper, Osterholm and coauthors ${ }^{11}$ concluded that recent expanded recommendations for influenza vaccination are based on expert and organizational opinion rather than on data. They also state that systematic overestimation of vaccine effectiveness has hampered the identification of better solutions, and they call for improved vaccine development based on novel antigens. ${ }^{11}$

In January 2013, the US Centers for Disease Control and Prevention analyzed the effectiveness of the 2012-2013 influenza vaccine; they concluded that this year's vaccine is only $55 \%$ effective against laboratory-confirmed influenza $\mathrm{A}$ and $70 \%$ effective against influenza B. ${ }^{12}$ They recommended that vaccinated individuals with influenza-like illness should be considered for antiviral treatment. ${ }^{12}$

Although expert opinion is a necessary element in making important policy decisions such as mandatory vaccination, these decisions should be driven primarily by the best available evidence. The aforementioned studies have highlighted major shortcomings with the current influenza vaccine that decision-makers should not ignore. Indeed, during the current season, a vaccinated health care worker may still be required to take antivirals during an outbreak, making receipt of the vaccine moot.

There is also uncertainty about influenzaassociated morbidity and mortality. The oftenquoted figures are worst-case scenarios derived from epidemiologic studies and mathematical models that estimate overall excess seasonal mortality because it is impossible to measure specific influenza outcomes on a population level. Whereas certainly some of these deaths are a result of the seasonality of influenza infection, many other respiratory viruses that can cause substantial illness (e.g., respiratory syncytial virus) circulate at the same time. These methodologies can only generate crude and broad estimates of influenza-related mortality.

Like others in our field, we get vaccinated against influenza each year, despite the vaccine's shortcomings, and we strongly encourage other health care workers to do the same. We are actively involved in our organization's annual influenza vaccination campaign. However, we are uncomfortable taking the next step of compelling vaccination given the considerable limitations of the current vaccine.

Mandatory vaccination, if implemented, may be legally challenged. This may lead to secondary challenges to other well-established mandatory vaccination efforts that have excellent data supporting their use. If a better vaccine and more robust literature about influenza-specific patient outcomes were available, the debate would be moot in favour of mandatory vaccination of health care workers. Unfortunately, important questions remain unanswered, and the endless debate about the utility of the current vaccine needs to stop. We need a better vaccine.

\section{References}

1. Lam P-P, Chambers LW, Pierrynowski MacDougall DM, et al Seasonal influenza vaccination campaigns for health care personnel: systematic review. CMAJ 2010;182:E542-8.

2. Bryce E, Embree J, Evans G, et al. AMMI Canada position paper: 2012 mandatory influenza immunization of health care workers. Can J Infect Dis Med Microbiol 2012;23:e93-5.

3. Flegel, K. Health care workers must protect patients from influenza by taking the annual vaccine. CMAJ 2013;184:1873.

4. Rakita RM, Hagar BA, Crome P, et al. Mandatory influenza vaccination of healthcare workers: a 5-year study. Infect Control Hosp Epidemiol 2010;31:881-8.

5. Schanzer DL, Tam TWS, Langley JM, et al. Influenza-attributable deaths, Canada 1990-1999. Epidemiol Infect 2007;135:1109-16.

6. Statement on seasonal influenza vaccine for 2011-2012. Ottawa (ON): Public Health Agency of Canada; 2011. Available: www.phac-aspc.gc.ca/publicat/ccdr-rmtc/11 vol37/acs-dcc-5/index -eng.php (accessed 2012 Dec. 4).

7. Thomas RE, Jefferson T, Lasserson TJ. Influenza vaccination for healthcare workers who work with the elderly. Cochrane Database Syst Rev 2010;(2):CD005187.

8. Osterholm MT, Kelley NS, Sommer A, et al. Efficacy and effectiveness of influenza vaccines: a systematic review and meta analysis. Lancet Infect Dis 2012;12:36-44.

9. Simonsen L, Taylor RJ, Viboud C, et al. Mortality benefits of influenza vaccination in elderly people: an ongoing controversy. Lancet Infect Dis 2007;7:658-66.

10. FluWatch. Ottawa (ON): Public Health Agency of Canada; 2013. Available: www.phac-aspc.gc.ca/fluwatch/index-eng.php (accessed 2013 Mar. 6).

11. Osterholm MT, Kelley NS, Manske JM, et al. The compelling need for game-changing influenza vaccines. An analysis of the influenza vaccine enterprise and recommendations for the future. Minneapolis (MN): Center for Infectious Disease Research \& Policy; 2012. Available: cidrap.umn.edu (accessed 2012 Dec. 4).

12. Early estimates of seasonal influenza vaccine effectiveness United States, January 2013. MMWR Morb Mortal Wkly Rep $2013 ; 62 ; 32-5$

Affiliations: From the Infection Prevention and Control Unit (Gardam, Lemieux), the Division of Infectious Diseases (Gardam) and the Division of Family Medicine (Lemieux), University Health Network; and the Faculty of Medicine (Gardam, Lemieux), University of Toronto, Toronto, Ont.

Contributors: Both of the authors contributed to the conception and design and to the drafting and revising of the article. Both of the authors approved the final version submitted for publication. 